\title{
Manuel Bamert
}

\section{„Aha!“ - Annotieren mit Stiften als epistemische Praxis}

\begin{abstract}
Reading notes in books and other printed matter are of increasing interest in Philology and Cultural History. However, we still lack an understanding of their epistemic foundations. With reference to Thomas Mann's private library, I suggest viewing the act of annotating with pens itself as an epistemic practice. For this, I introduce the term 'pen traces' for all phenomena left behind by pens, and distinguish between four different forms of knowledge that influence their emergence: the knowledge of readers, the knowledge of authors, textual knowledge and contextual knowledge. By shedding light on pen traces as traces of an epistemic practice, finally, I point out the need for a practice theory of annotation.
\end{abstract}

Keywords: Practice Theory, Role of Interpretation, Commenting, Highlighting, Form, Cultural Anthropology, Epistemology, Literature

\section{Einleitung}

Als Thomas Mann 1938 an seinem Roman Lotte in Weimar arbeitete, war sein Schreibprozess, wie für ihn üblich, von umfangreichen Lektüren begleitet. So las er zu dieser Zeit beispielsweise das im gleichen Jahr neu erschienene Buch des Orientalisten Hans Heinrich Schaeder mit dem Titel Goethes Erlebnis des Ostens. Das Buch, das in der Nachlassbibliothek im Thomas-Mann-Archiv der ETH Zürich erhalten ist, hat Mann indes nicht nur gelesen - er hat es, wie er es an anderer Stelle nennt, „mit dem Bleistift“ gelesen. ${ }^{1}$ Will heißen: Er hat darin zahlreiche Anund Unterstreichungen, Ausrufezeichen und Marginalien hinterlassen. An einer Stelle etwa setzt Mann ein „Aha!“ (siehe Abbildung 1 auf Seite 22) an den Rand des Textes und legt damit nahe, dass er hier eine Erkenntnis gewonnen hat. ${ }^{2}$

1 Wiederholt dokumentiert Mann in seinen Tagebüchern Lektüren auf diese Weise, so zum Beispiel am 18. Mai 1919, am 31. Mai 1921 (siehe Mann 1979, 240, 524) und am 24. Februar 1946 (siehe Mann 1986, 310).

2 Die entsprechende Marginalie findet sich in Schaeder (1938, 30). Alle Abbildungen in diesem Beitrag entstammen der digitalen Datenbank zu Manns Nachlassbibliothek, der Abdruck erfolgt mit freundlicher Genehmigung des Thomas-Mann-Archivs der ETH Zürich. Siehe zu Abbildung 1 Datenbank Thomas Mann Nachlassbibliothek, Thomas Mann 552, Bild 47 (abgerufen am 14.10.2019). 
Rund um diese Phänomene, also um all die mit Stiften in Büchern und anderen Schriften hinterlassenen Lesespuren, hat sich inzwischen eine eigene Disziplin in einem „kulturhistorisch-philologischen Spannungsfeld“ gebildet (Moulin 2010, 20). Die Lesespurenforschung, die ihre wissenschaftshistorischen Wurzeln in der Untersuchung mittelalterlicher Glossen hat (Moulin 2010, 20), dringt in jüngster Zeit vermehrt auch in den digitalen Raum vor, wie etwa die Erschließungs- und Digitalisierungsprojekte zu Thomas Manns (Thomas-Mann-Archiv der ETH Zürich 2019), Herman Melvilles (Olsen-Smith et al. 2008), Theodor Fontanes (TheodorFontane-Archiv Potsdam und UCLAB an der Fachhochschule Potsdam 2019) oder Jacques Derridas (Center for Digital Humanities at Princeton 2018) Lesespuren zeigen.

Theoretisch unterbeleuchtet sind auf diesem Feld derweil noch die epistemischen Grundlagen solcher Lesespuren. Welcher Wissensgehalt kommt mit Stiften vorgenommenen Annotationen wie dem randständigen „Aha!“ zu? Dieser Frage möchte ich im Folgenden anhand von einigen Beispielen aus Thomas Manns Nachlassbibliothek nachgehen. Dazu werde ich zunächst den Begriff der Annotation im Kontext der Lesespurenforschung situieren. Danach nehme ich eine epistemologische Perspektive ein und zeige in Ansätzen auf, wie man die vorgebrachten Phänomene als Spuren epistemischer Praktiken konzeptualisieren kann. Zuletzt werde ich unter dem Stichwort Praxeologie darauf eingehen, dass eine praxistheoretische Analyse des Annotierens ein größeres Forschungsdesiderat ist.

\section{Terminologie}

Anders als in der Glossenforschung, die sich um eine einigermaßen etablierte Terminologie bemüht hat (vgl. dazu zum Beispiel Bergmann und Stricker 2009), ist die Lage in der Erschließung und Erforschung von neuzeitlichen Lesespuren typologisch und terminologisch ungeklärt. In der Einleitung zum in der deutschsprachigen Lesespurenforschung bislang umfangreichsten Sammelband mit dem Titel Lesespuren - Spurenlesen verwendet Marcel Atze etwa alleine für durch Schreibwerkzeuge entstandene Lesespuren über 20 verschiedene Begriffe. In der Reihenfolge ihres Erscheinens im Text sind das: „Bearbeitungsspuren“, „Kommentare“, „Korrekturen“, „Nachträge[]“, „Zusätze[]“, „Ergänzungen“, „Eintragungen“, „Anmerkungen“, „Lesespuren“, „Marginalien“, „Phänomen der Handschrift im Buch“, „Annotationen“, „Randnotizen“, „Einträge“, „Anstreichungen“, „Randglossen“, „Randschriften“, „graphische Merkzeichen“, „Randmarkierungen“, „Notizen von des Dichters Hand“, „Randbemerkungen“, „Änderungen“, „Aufzeichnungen“ und schließlich „Lektürespuren“. Diese substantivischen Begriffe werden bei Atze 
zudem oft kombiniert mit Verben wie ,,annotier[en]“ und Adjektiven wie „eigenhändig“ oder „handschriftlich“. ${ }^{3}$ Das Begriffsfeld für die Beschäftigung mit Lesespuren ist also bereits breit abgesteckt. Was hingegen fehlt, sind klare Definitionen, die die untersuchten Phänomene systematisch charakterisieren und voneinander abgrenzen.

Der Begriff der Annotation ist auf diesem Feld nur einer von vielen und sein Geltungsbereich ist unklar. Klar ist einzig, dass er nicht als Synonym zum Begriff der Lesespur taugt, denn terminologisch konsequent müssen auch Eselsohren und schiefe Buchrücken als Lesespuren gelten - als Annotationen würde diese aber wohl kaum jemand bezeichnen. Wie lässt sich der Begriff der Annotation im Hinblick auf Lesespuren also charakterisieren? Wo sind seine Grenzen?

Etymologisch geht die Annotation auf das Lateinische zurück. Das Wort setzt sich zusammen aus dem Präfix ad- und dem Verb notare, welches wiederum aus dem Substantiv nota für „Kenn-, Merk-, Schrift-, Tonzeichen, Anmerkung“ (siehe den Eintrag ,Note“ in Etymologisches Wörterbuch des Deutschen 1993) beziehungsweise „Kennzeichen, Zeichen, Merkmal, Schrift, typographische Zeichen“ (siehe den Eintrag ,notieren' in Etymologisches Wörterbuch der deutschen Sprache 2011) abgeleitet ist (vgl. auch den Eintrag ,annotate' in The Concise Oxford Dictionary of English Etymology 1996). Auch im Deutschen, Englischen und Französischen noch klingt der Begriff der Annotation an die Verben notieren, to note oder noter an und befindet sich damit in der Nähe von schreiben. ${ }^{4}$ Implizit wird er denn auch oft im Sinne schriftlicher Lesespuren verstanden, zum Beispiel im Kommentierten Katalog der nachgelassenen Bibliothek von Johannes Bobrowski, wo Dalia Bukauskaite Annotationen als „Randbemerkungen Bobrowskis zum Text“ (Bukauskaitė 2006, XXXIX) definiert. Auch im Digitalisierungsprojekt zu Herman Melvilles Bibliothek werden „annotations“ auf schriftliche Phänomene beschränkt, da sie von „markings" unterschieden werden; es heißt dort jeweils, ein bestimmtes Exemplar sei „marked and annotated“ (Olsen-Smith et al. 2008).

Gar keine Rolle spielt das Schriftkriterium in Bezug auf den Begriff der Annotation hingegen im neueren Digitalisierungsprojekt Derrida's Margins. Die Annotation ist dort kein unter-, sondern ein übergeordneter Terminus. So gelten auch jene Seiten als „annotated pages“, die zum Beispiel nur Anstreichungen aufweisen, und die Facette „Annotation Type“ in der Suchfunktion umfasst Auswahlmöglichkeiten wie „underlining“, „circling“, „arrow“ und explizit auch „,nonverbal annotation“ (Center for Digital Humanities at Princeton 2018).

3 Aufgeführt werden hier nur die Begriffe, die Atze selbst verwendet; dazu kämen noch weitere, die er aus anderen Texten zitiert, vgl. Atze (2011).

4 Vgl. dazu auch Fischer (1997, 99), der Notation für einen „neutraleren“ Terminus als Schrift hält. 


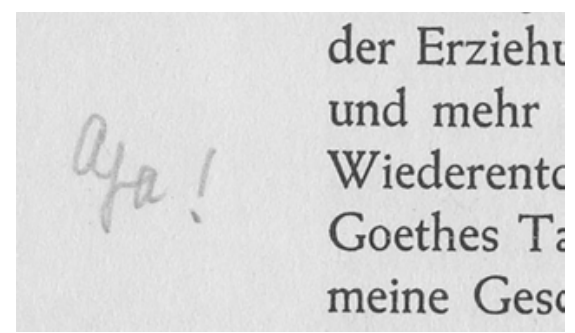

Abb. 1: Manns ,Aha!'-Marginalie in Schaeders Goethes Erlebnis des Ostens genau auf den Zeitpunkt, zu itrat. 1783 fand er ihn in der 5 'Reise nach Ostindien und iff der Ballade 'Der Gott und trialegende war freilich schon leisewerke des holländischen Wetzlar las und im zwölften t. Aber sie weicht dort von

Abb. 2: Ausrufezeichen, Anstreichung und Unterstreichungen im selben Exemplar

Während sich die eingangs erwähnte ,Aha!'-Marginalie also ohne Weiteres als Annotation bezeichnen lässt, ist der Fall bei den Lesespuren auf Abbildung 2 schon etwas unklarer. ${ }^{5} \mathrm{Um}$ Schrift handelt es sich hier zumindest bei den An- und Unterstreichungen nicht mehr - aber noch um Annotationen? Und wie verhielte es sich mit den Spuren von Textmarkern, die nichts Anderes als farbige Markierungen sind? Lassen sich auch diese Phänomene als Annotationen bezeichnen? $\mathrm{Zu}$ solchen Fragen finden sich in verschiedenen wissenschaftlichen Disziplinen widersprüchliche Ansichten, und zwar auch innerhalb der jeweiligen Disziplin.

In den 1990er und 2000er Jahren haben sich beispielsweise verschiedene Forschungsgruppen aus den Human-Computer Studies Gedanken darüber gemacht, wie man das Annotieren auf Papier in digitale Techniken überführen kann. Interessant sind diese Bemühungen aus Sicht der Lesespurenforschung, weil die Forschenden dazu naturgemäß erst das Annotieren selbst beziehungsweise die Materialisierung der damit verbundenen Praktiken konzeptualisieren mussten. Und tatsächlich zeigten sich hierbei ähnliche Differenzen, wie sie auch in der stärker geisteswissenschaftlich ausgerichteten Lesespurenforschung zu finden sind. Während etwa die Gruppe um Ilia Ovsiannikov bloße Markierungen ebenfalls als Annotationen bezeichnete (Ovsiannikov et al. 1999, 335), distanzierten sich Daniela Fogli et al. ausdrücklich von dieser Auffassung:

On the whole, a human creates an annotation using a set of graphical elements which s/he considers meaningful (letters, elements of an alphabet, icons). S/he uses visual identifiers and visual links whenever s/he considers necessary to make the link between annotation and base clearer. This stance is different for example from the one in [Ovsiannikov et al.], where marking is considered as a form of annotation and visual linking not explicitly considered. (Fogli et al. 2004, 98)

5 Abbildung 2 zeigt einen Ausschnitt aus Schaeder (1938, 141), siehe Datenbank Thomas Mann Nachlassbibliothek, Thomas Mann 552, Bild 158 (abgerufen am 14.10.2019). 
Wo die Grenzen des Annotationsbegriffs verlaufen (sollen), ergibt sich also auch in dieser Forschungsperspektive nicht von selbst, sondern ist Gegenstand von definitorischen Aushandlungen und variiert je nach Anwendungskontext.

Gemeinsam ist den unterschiedlichen Annotationskonzepten auf dem gesamten Feld zwischen Informatik, Kognitionswissenschaft und Linguistik derweil ihre funktionalistische Ausrichtung. Annotationen werden im dortigen Verständnis immer intentional und zweckgerichtet angelegt. Der Annotationsbegriff folgt auf diesem Feld - bei aller konzeptionellen Diversität - einer Verengung seines etymologischen Gehalts: Annotieren heißt in diesem Sinn nicht nur Zeichen produzieren, sondern Zeichen zu einem bestimmten Zweck produzieren.

Auf diesem weiten Feld außerhalb dessen, was ich im engeren Sinn als Lesespurenforschung bezeichne, wurde von verschiedener Seite vorgeschlagen, den Begriff der Annotation über die Funktion der Hinzufügung zu einem Text zu definieren. So wird der Begriff der Annotation zum Beispiel im Glossar des Projekts forTEXT wie folgt beschrieben: „Die manuelle oder automatische Hinzufügung von Zusatzinformationen zu einem Text. Bereits eine Kursivierung oder Fettung können in einem weiten Begriffsgebrauch als Annotationen bezeichnet werden.“ (Meister 2016, Hervorhebungen im Original).

Typisch für solche Definitionen ist die implizite Unterscheidung von Annotationsbasis (in diesem Fall ein Text) und der eigentlichen Annotation, der immer die Form eines Zusatzes, die Eigenschaft der Sekundarität eignet. Bemerkenswert ist indes, wo das Glossar von forTEXT die Grenze zwischen der Basis und der Sekundarität zieht: Wird ein Text in seiner konkreten Erscheinung modifiziert, kann das im weiten Begriffsgebrauch bereits eine Annotation sein.

Überträgt man dieses Begriffsverständnis auf das Feld der Lesespurenforschung, dann sind also auch die Phänomene auf Abbildung 2 auf der vorherigen Seite Annotationen. Doch auch dieser weite Begriffsgebrauch, der selbst das umfasst, was die Typographie Auszeichnung nennt, lässt sich mit Grenzfällen konfrontieren, etwa einer Streichung wie auf Abbildung 3 auf der nächsten Seite. ${ }^{6}$ Ist auch diese Lesespur eine Hinzufügung von Zusatzinformation? Oder ist es eher eine Informationsmodifikation und damit allenfalls keine Annotation? Aus philologischer Sicht ist das keine einfache Frage und ihre Beantwortung hängt von der jeweiligen Perspektivierung ab.

Eine Hinzufügung ist das gezeigte Phänomen nur, wenn man die spezifisch materielle Konstellation berücksichtigt, die hier vorliegt. Hätte Thomas Mann die Möglichkeit gehabt, den gezeigten Text in digitaler Form zu lesen, hätte er das

6 Abbildung 3 zeigt einen Ausschnitt aus Mann (1954, 100), siehe Datenbank Thomas Mann Nachlassbibliothek, Thomas Mann 114, Bild 105 (abgerufen am 14.10.2019). 
TONIO KRÖGER

1903

Die Wintersonne stand nur als armer Schein, milchig und matt hinter Wolkenschichten über der engen Stadt. Naß und
Zuqig Art von weichem Hagel, nicht Eis, nicht Schnee.

Abb. 3: Mann korrigiert eine gedruckte Ausgabe seiner Erzählung Tonio Kröger

gestrichene Wort möglicherweise einfach gelöscht und durch dasjenige ersetzt, das er hier neben der Zeile eingefügt hat. Hier haben wir es aber mit einer anderen materiellen Konstellation zu tun, denn es handelt sich um einen gedruckten Text, der sekundär mit einem Stift bearbeitet wurde. Materiell gesehen wurde hier nichts ersetzt, sondern dem gedruckten Text wurde, wenn man so will, die Information hinzugefügt, dass da ein Stift am Werk war.

Damit ist denn auch das Gemeinsame aller bisher gezeigten Phänomene benannt - es ist das Medium ihrer Produktion beziehungsweise die daraus resultierende Materialität: Es handelt sich um die Spuren von Stiften. Und um den zentralen materiellen Aspekt dieser bestimmten Art von Annotationen hervorzuheben, nenne ich diese Phänomene Stiftspuren.

\section{Epistemologie}

Wie das in Thomas Manns Nachlassbibliothek erhaltene Material zeigt, hat Mann vor allem in Sachtexten Stiftspuren hinterlassen - also eben in Texten wie Schaeders Goethes Erlebnis des Ostens. Literarische Texte hingegen, auch solche, von denen bekannt ist, dass er sie gelesen hat (und zwar in der jeweils vorliegenden überlieferten Ausgabe), weisen oft wenige bis gar keine Stiftspuren auf (vgl. dazu Bamert, in Vorbereitung).

Ein ebenso einfacher wie zentraler Grund für die Lektüre eines Textes kann sein, dass dieser Wissen bereithält, über das sich die lesende Person kundig machen will. Ich möchte eine solche Lektüreweise in der Folge epistemisch nennen und sie heuristisch von anderen, zum Beispiel ästhetischen Lektüren, unterscheiden. In einer epistemischen Lektüre wird ein Text demnach als Wissensträger rezipiert, in einer ästhetischen Lektüre hingegen wird der Text vorrangig als Kunst- 
werk wahrgenommen. Diese Unterscheidung ist freilich nicht dichotomisch oder antagonistisch zu verstehen, sondern im Sinne zweier Prinzipien, die auch in eins fallen oder sich gegenseitig ergänzen oder überlagern können.

Die Unterscheidung zwischen epistemischen und ästhetischen Lektüren zielt also auf unterschiedliche Zugangsweisen zu einem Text und nicht auf die Charakterisierung des Textes selbst. Jeder Text kann Gegenstand unterschiedlichster Lektüreformen werden. Und doch stehen die Beschaffenheit eines Textes und der Zugang zu diesem in einem wechselseitigen Wirkungsverhältnis, das erst recht intrikat wird, wenn man noch weitere Faktoren hinzuzieht. Erstens gibt es empirisch beschreibbare Textualitätsmuster, aufgrund derer man einen Text einer Textsorte zurechnen kann, zweitens ist da das lesende Subjekt, das diesen Bezug auf je eigene Weise vollzieht, drittens gilt es die Instanz des Autors beziehungsweise der Autorin zu berücksichtigen, die die Rezeption nicht zuletzt auch paratextuell beeinflusst, und viertens existieren noch vielfältige weitere lektüreregulierende Aspekte, die man unter dem Sammelbegriff der Kontexte zusammenfassen kann. ${ }^{7}$

Geht man nun davon aus, dass Textsorten innerhalb dieses Gefüges eine kommunikative Funktion erfüllen (vgl. dazu Hausendorf et al. 2017, 319), dass zudem gewisse Texte musterhaft der kommunikativen Aufgabe der Wissensvermittlung dienen und beobachtet man schließlich, dass vorzugsweise gerade diese Texte annotiert werden, dann hat man hierin einen ersten Hinweis darauf, dass das Lesen mit dem Stift unter bestimmten Umständen eine Fortführung der epistemischen Lektüre mit dinglichen Mitteln sein könnte. Das Lesen mit dem Stift wäre dann selbst als epistemische Praxis zu beschreiben.

In kognitionswissenschaftlich ausgerichteten Forschungsansätzen ist diese Sichtweise jedenfalls bereits verbreitet, wenn auch mittels anderen Vokabulars. Kenton P. O'Hara et al. beispielsweise, die sich der Readers-as-Writers-Thematik aus der Perspektive der Human-Computer Studies annahmen, haben dem Annotieren während des Lesens vielfältige Funktionen zugeschrieben und es explizit mit dem Wissen der Lesenden in Verbindung gesetzt:

Annotations help engage the writer with the source materials supporting a whole range of activities such as navigation, linking information across source materials and between source materials and composition, structuring thoughts, offloading new idea threads to be followed later on in the composition and offloading the results of tasks taking place across multiple documents. The annotations used during these tasks were not stand-alone but rather were

7 Die vier aufgeführten Kategorien gehen auf das Modell der literarischen Kommunikation zurück, vgl. Köppe (2011, 2). Unter Kontexte seien all jene für das literarische Feld relevanten Faktoren zusammengefasst, die durch die Text-, Leser/Leserin- und Autor/Autorin-Instanz nicht abgedeckt sind, zum Beispiel weitere Institutionen des Literaturbetriebs oder gesellschaftliche Verhältnisse und Diskurse. 
pointers to knowledge remembered, or represented internally, by the author. (O'Hara et al. 2002, 298)

Das Annotieren wird hier also als Hilfsmittel zur Orientierung, Informationsverknüpfung, Strukturierung und kognitiven Entlastung während des Lesens beschrieben und die resultierende Annotation als Hinweis auf das Wissen der lesenden Person verstanden. Noch stärker systematisiert findet sich diese Sichtweise in der Unterscheidung zwischen kognitiven Organisations- und Elaborationsstrategien, wie sie Philipp Maik dargelegt hat. Als kognitive Organisationsstrategien bezeichnet Maik diejenigen Techniken, die Lesenden dabei helfen, „die Struktur des Texts und der in ihm enthaltenen Informationen zu erkennen“ (Maik 2015, 215). Die Organisationsstrategien werden von Lesenden, die auf einen Wissenstransfer aus sind, angewandt, um Texte beziehungsweise deren Elemente so zu (re)konfigurieren, dass sie sich in die eigenen mentalen Prozesse einpassen lassen - was explizit auch Stiftgebrauch umfassen kann: „Beispiele für diese Form der Strategien sind das Unterstreichen von zentralen Begriffen, das Anfertigen von Zusammenfassungen, Notizen und Schaubildern oder auch die Darstellung einer Argumentationsstruktur.“ (Maik 2015, 215).

Dass es sich dabei um ein Zusammenspiel von Textwissen und Lesendenwissen handelt, wird noch klarer bei der Beschreibung der kognitiven Elaborationsstrategien, bei denen es darum geht, „über die eigentlichen Textinhalte hinauszugehen (sie zu elaborieren), um die Textinhalte ins Langzeitgedächtnis zu integrieren“ (Maik 2015, 215). In der Verarbeitung der Informationen, die der Text bereithält, wirkt das Lesendenwissen hier explizit als gestaltende Kraft, denn wie die Elaboration des Textwissens erfolgt, hängt maßgeblich davon ab: Eine lesende „Person aktiviert beispielsweise ihr Vorwissen vor dem Lesen, stellt offene Fragen an den Text, kommentiert ihn, klärt unbekannte Wörter oder versucht, den weiteren Textinhalt zu prognostizieren.“ (Maik 2015, 215).

Aus kognitionswissenschaftlicher Sicht scheinen Wissensbestände den Stifteinsatz während der Lektüre also in zweifacher Form zu bestimmen: Wie sich schon in der textsortenspezifischen Lesespurenverteilung andeutet, führt einerseits insbesondere das in den gelesenen Büchern vorgefundene Wissen zu Markierungen und Marginalien durch Stifte. Vor diesem Hintergrund kann man etwa Unterstreichungen als Segmentierung eines Textes in einzelne Informationen verstehen und Pfeile, Umrahmungen, Farbmarkierungen und Nummerierungen dienen entsprechend der komplexeren Darstellung von Informationsstrukturen und -relationen. In dieselbe Kategorie lassen sich jene Marginalien einordnen, die am Rand des gedruckten Textes dessen Inhalte in Stichworten zusammenfassen und so das 
Textwissen zugänglicher machen sollen. ${ }^{8}$ Andererseits spielt auch das durch die Lektüre angeregte Wissen der Lesenden eine Rolle und wird in Stiftspuren sichtbar, zum Beispiel durch Kommentare, die auf das in den Drucktexten repräsentierte Wissen eingehen und es mit eigenem Wissen konfrontieren. In der Lektüre mit dem Stift reagiert demnach das Wissen beziehungsweise das Nichtwissen der Lesenden mit dem Wissen beziehungsweise Nichtwissen der Texte.

Doch an diesem Punkt darf man nicht stehen bleiben. Man sollte vielmehr stets davon ausgehen, dass weitaus mehr Wissensformen und -bestände an einem Lektüreprozess beteiligt sind als nur ein Textwissen und das Wissen der lesenden Person. Auch hier möchte ich eine heuristische Abstraktion anregen und angelehnt an das Modell der literarischen Kommunikation (vgl. dazu Köppe 2011, 2, bzw. Fn. 7) vorschlagen, in die Lesespurenforschung mindestens vier Wissenskategorien einzubeziehen: neben dem Textwissen (T-Wissen) und dem Wissen der lesenden Person (L-Wissen) auch das Wissen der Autoren beziehungsweise Autorinnen (A-Wissen) und das Kontextwissen (K-Wissen).

Es versteht sich von selbst, dass eine solche Abstraktion enorm divergierende Wissenskonzepte impliziert. Doch kann gerade die Ausarbeitung der jeweiligen Divergenzen - im Sinne einer Heuristik eben - produktive Effekte zeitigen. In dem Moment, in dem man neben dem T-Wissen und dem L-Wissen auch ein A-Wissen und ein K-Wissen in Betracht zieht, öffnet sich der Blick für epistemische Aspekte von Lesespuren, die vorher notwendigerweise unbeachtet blieben. So, wie man einen (literarischen) Text je nach theoretischem und methodischem Ansatz aus unterschiedlichen Perspektiven analysieren kann (im Hinblick auf seine textuelle Eigenlogik, auf die Rezeption durch Lesende, als Produkt eines Autors beziehungsweise einer Autorin oder auf die ihn beeinflussenden Kontexte), so kann man in der epistemologischen Analyse auch Lesespuren unterschiedlich perspektivieren und sie hinsichtlich des T-Wissens, des L-Wissens, des A-Wissens oder des K-Wissens untersuchen.

Reduziert man die Lesespurenforschung hingegen auf die Beziehung zwischen T-Wissen und L-Wissen, schließt man schlicht bestimmte Wissensformen und -bestände aus und generiert damit Blindstellen. Zwar scheint die Reduktion auf das Zusammenspiel von T-Wissen und L-Wissen oft naheliegend, da die anderen Wissensinstanzen nur abstrakt vorliegen. Eine Marginalie zum Beispiel, deren Referenz offenkundig ist, verweist vermeintlich direkt auf das Textwissen, das zu ihrer Entstehung angeregt hat, und auf das Wissen der lesenden Person, das sich

8 Solche Marginalien scheinen in funktionaler Hinsicht ihre gedruckten Verwandten zu imitieren, denn auch gedruckte Marginalien „schaffen durch Ergänzungen wie Definitionen, Kommentare, Zitate und Verweise einen Zusatznutzen für den Leser“, siehe Huse (2015). 
in der Marginalie auf die eine oder die andere Weise manifestiert hat. Das K-Wissen und das A-Wissen treten hinter diese scheinbar unmittelbaren Wissensformen zurück und es leuchtet ein, dass sie nur durch indirekte analytische Verfahren ermittelt werden können.

Bei genauerem Hinsehen gilt genau letzteres aber für alle Wissensinstanzen, denn es gibt keine Wissensform, deren Beteiligung an der Entstehung von Lesespuren sich unmittelbar zeigt. Auch die simpelsten Lesespurphänomene setzen interpretative Verfahren voraus, will man ihre Bedeutung ermessen und das Wissen rekonstruieren, das zu ihrer Manifestation geführt hat. Selbst die einförmigste Markierung ergibt sich nicht einfach von alleine aus dem Text oder aus Kopf und Hand der lesenden Person, sondern immer aus einem sozial regulierten und gleichzeitig situativ modulierten Zusammenspiel mehrerer Instanzen, und jede dieser Instanzen ist mit ihren je spezifischen Wissensbeständen in Betracht zu ziehen. Welche Informationen jemand für wichtig - mit anderen Worten: wissenswert erachtet, hängt von vielen Faktoren ab. Das epistemologische Erkenntnispotential von Lesespuren liegt demnach nicht nur darin, dass sie Spuren des Wissens von Lesenden und Gelesenem sind. Sie sind auch Spuren des Wissens der Autoren oder Autorinnen und der Kontexte ihrer Entstehung - und der jeweiligen Relationen dieser Wissensformen und -inhalte zueinander.

Ich komme damit nochmals auf Manns vermeintliches Aha-Erlebnis zurück. In einem Aha-Moment zeigt sich - normalerweise - so etwas wie ein Erkenntnismoment, man könnte auch sagen: das Verschwinden eines Nichtwissens. Betrachtet man die ,Aha!'-Marginalie isoliert, könnte man darin demnach Manns Nichtwissen erkennen, dem angesichts eines T-Wissens ein Erkenntnismoment widerfährt. Tatsächlich verhält es sich in diesem Fall jedoch genau umgekehrt. Das ,Aha!' ist hier Ausdruck eines L-Wissens, das dem T-Wissen überlegen ist. Man muss Manns ,Aha!‘ im Kontext aller Marginalien lesen, die er in Schaeders Buch hinterlassen hat. Sie lauten zum Beispiel: „Dummkopf“, „Pfui!“”, „Unverschämt!“ sowie „Schafskopf und Fälscher“ (siehe Abbildungen 4 und 5 auf der nächsten Seite). ${ }^{9}$

Manns Ärger wird verständlich, wenn man sich mit Schaeders Buch auseinandersetzt. So heißt es im 2003 erschienenen Kommentarband zur Neuedition von Lotte in Weimar über Schaeders Buch:

Der Berliner Orientalist liest Goethe aus dem Geiste der nationalsozialistischen Bewegung und hat seine liebe Mühe, gerade dessen Hinwendung zum Osten, zur orientalischen Dichtung und Philosophie, wie auch seine Haltung in der Zeit der vaterländischen Begeisterung

9 Die Marginalien „Dummkopf“ und „Unverschämt!“ finden sich in Schaeder (1938, 14, 20), Abbildungen 4 und 5 zeigen Ausschnitte aus Schaeder (1938, 19, 46). Siehe Datenbank Thomas Mann Nachlassbibliothek, Thomas Mann 552, Bilder 31, 36, 37, 63 (abgerufen am 14.10.2019). 


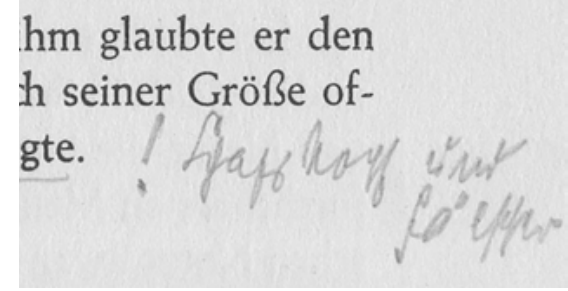

Abb. 4: „Schafskopf und Fälscher“

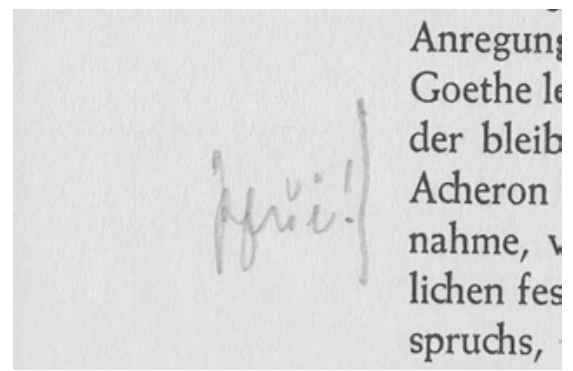

Abb. 5: „Pfui!“

so hinzubiegen, dass sie 1938 politisch korrekt wirken. ,Fälscher‘ nennt ihn Thomas Mann deshalb zu Recht und mit besserem Wissen, wenn Schaeder behauptet, die ,deutsche Erhebung‘ von 1813 habe Goethes ,Gemüt‘ bewegt. (Mann 2003, 114. Keine Hervorhebung im Original)

So wichtig das Buch als Quellenliteratur für Thomas Mann auch war, so deutlich war seine Abneigung gegen das „deutsch-völkisch orientierte Buch“ (Mann 2003, 114). Seine Lektüre beschrieb Mann in seinem Tagebuch als „ärgerlich, aber interessant“. ${ }^{10}$ Mit besserem Wissen also liest Thomas Mann seinen Schaeder und in diesem Sinn muss wiederum sein ,Aha!' gelesen werden. Mann markiert hier keine Erkenntnis, vielmehr markiert er in ironischer Weise gerade die Absenz eines Erkenntnismoments. Auf diese Weise macht er die für das Verhältnis zwischen Sachtext und lesender Person eigentlich geltende Hierarchie transparent - und offenbart in dieser Geste ein weiteres, implizites Wissen über die zu erwartende Hierarchie zwischen T-Wissen und L-Wissen: Normalerweise müssten Sachtexte dem Wissen derjenigen, die sie lesen, überlegen sein.

In diesem Fall ist das anders (zumindest stellenweise, denn Mann hat durchaus ausgiebig Wissen aus Schaeders Buch verarbeitet). Mann selbst zieht eine weitere Instanz in die Pflicht, indem er nicht nur auf den Text zielt, sondern dessen Autor kritisiert und diesem als Fälscher die Verbreitung von Falschwissen vorwirft. Worauf man, aus der heutigen, historisierenden Perspektive schließlich, die ,Aha! ‘Marginalie als Spur eines Kampfes um die Etablierung des richtigen Wissens sehen kann, das dereinst in der Form historischer Tatsachen zum K-Wissen werden sollte.

Das Beispiel zeigt, dass bei einer Lektüre auch andere Relationen möglich sind als nur diejenige zwischen Text und lesender Person, wie es funktional rationalisierte Modelle nahelegen. Und es zeigt überdies, dass Annotationen auch in weltanschaulicher Hinsicht zu verstehen sind. Der kognitionswissenschaftliche

10 Tagebucheintrag vom 28. Juli 1938, siehe Mann (1980, 262). 
Befund, dass Annotationen dazu dienen, Lesende kognitiv zu entlasten und Material zu strukturieren, ist eine hilfreiche Standardformel. Doch wer mit Stiften liest, kann noch mehr wollen, zum Beispiel korrigieren und kommunizieren - mithin während des Lesens bereits schreiben. Gerade die Fälle, in denen Stiftspuren in den bestehenden Text eingreifen, sind aus philologisch-hermeneutischer Sicht höchst komplex, weil das Lesen dabei tatsächlich übergangslos in ein Schreiben kippen kann. Auch dieses Verhältnis lässt sich freilich wieder als Wissensrelation verstehen, wenn nämlich ein T-Wissen mit einem überlegenen L-Wissen konfrontiert und überschrieben wird.

\section{Praxeologie}

Ich habe skizziert, was ich meine, wenn ich das Lesen mit dem Stift als epistemische Praxis bezeichne. Unterbestimmt ist jedoch noch, worin hierbei der Praxisaspekt besteht - und ich halte das für ein größeres, die Möglichkeiten meines Beitrags übersteigendes Desiderat. Was der Lesespuren- beziehungsweise der Annotationsforschung fehlt, ist eine ausgereifte Praxistheorie auf der Grundlage von Detailstudien des Annotierens. Eine Theorie also, die nicht nur nach bestimmten Funktionen von Handlungen fragt, sondern nach den historischen und kulturellen Bedingungen, unter denen diese Handlungen realisiert werden. Erst dann nämlich werden die Handlungen ,als an impliziten Normen orientierte Handlungsroutinen“ (Martus und Spoerhase 2009, 89) und somit als Praktiken erkennbar, die ansonsten allzu oft theoretisch unbeachtet bleiben (vgl. dazu auch Wolff 2008).

Konfrontiert man die kognitionswissenschaftliche Sichtweise auf Annotationen mit einer kulturhistorischen Perspektive, präsentiert sich die Sachlage notwendigerweise komplex, da weitere Ebenen in die Analyse von Lesespuren einbezogen werden müssen. Der rein funktionale Erklärungsansatz wird in dieser Perspektive durch den Imperativ der gesellschaftlichen und zeitlichen Situierung herausgefordert. Annotationen sind demnach nicht nur als Manifestation kognitiver Prozesse $\mathrm{zu}$ verstehen. Annotationen sind die praktische Umsetzung des sozial regulierten Wissens vom epistemischen Potential der Stifte.

So müsste eine Praxeologie etwa nach den körperlichen Aspekten des Annotierens fragen. Welche Rolle wird der Körperhaltung und bestimmten Körperteilen für je unterschiedliche Lektüreformen und den ihnen entsprechenden Annotationspraktiken beigemessen? Welches inkorporierte Praxiswissen steckt möglicherweise in welchen Annotationsformen? Vielleicht könnte man damit noch besser in den Blick bekommen, warum sich bei Annotationen mit Stiften die Strichführung oftmals nicht nur im Fall von schriftlichen Annotationen, sondern sogar von An- und 
Unterstreichungen einer bestimmten Person zuordnen lassen, warum also selbst einfachste Annotationen einen eigenen Duktus aufweisen. Bei gewissen Annotationspraktiken ist der körperliche Anteil jedenfalls im Wortsinn nicht von der Hand zu weisen (nicht umsonst spricht auch die Editionsphilologie in metonymischer Weise von der Hand, wenn sie die Person meint).

Eine solche Praxeologie müsste des Weiteren nach den situationellen Aspekten des Annotierens fragen und beschreiben, unter welchen Umständen annotiert wird, beziehungsweise in welche Situationen sich eine lesende Person bringen muss, um annotieren, also in dieser spezifischen Weise epistemisch tätig sein zu können. Welche Medien(-konstellationen) erlauben mobile Lektüren und Annotationspraktiken on the go? Welche Stifte werden zu einer bestimmten Zeit wem und von wem aus welchen Gründen für welche Situationen empfohlen? Nicht zuletzt könnte man durch die Integration dieser praxeologischen Perspektiven das Lesen mit dem Stift und das Annotieren in neuartiger Weise mit den verwandten Praktiken des Exzerpierens, Notizenmachens und Schreibens ${ }^{11}$ abgleichen und dabei schärfen.

Methodisch müsste sich eine Praxeologie des Annotierens gleichermaßen diskursive Wissensformen über die Praktiken wie auch die Spuren der Praktiken selbst vornehmen, und so auch dem impliziten und praktischen Wissen vom Annotieren nachspüren. Wie und wo lernt man etwa die Praktiken und die Tabus des Stiftgebarens in Büchern? Gerade bezüglich des Praxiswissens, das man an der Schnittstelle zwischen Epistemologie und Praxeologie verorten muss, sind von einer zukünftigen Lesespurenforschung noch weitere Erkenntnisse zu erwarten. Denn es ist in erster Linie dieses Wissen, über das wir oftmals nur vor- oder unbewusst verfügen und das wir deswegen gar nicht als solches wahrnehmen.

Vor allem aber müssten praxeologische Studien den kommunikativen Aspekten des Annotierens nachgehen. Diese Perspektive würde helfen, Annotationen über ihr Dasein als Spuren epistemischer Praktiken hinaus auch als kommunikative Boten zu verstehen. ${ }^{12}$ In diesem Verständnis erkennt man die Annotationen also nicht mehr nur als Rückstand von etwas Vergangenem an, sondern in actu als Realisierung von Kommunikationspraktiken. Im Bereich der Lesespuren ist hier zum Beispiel an die Marginalien zu denken, die als Lob oder als Beleidigungen am Rande daherkommen. Aber auch in anderen Umgebungen lassen sich viele Annotationen als spezifische Kommunikationsformen verstehen, beispielsweise als Kommentare, ein genuin kommunikatives Phänomen also.

11 Vgl. dazu den Beitrag von McCarty (2020, in diesem Band).

12 Vgl. zur Unterscheidung von Spuren und Boten Krämer (2016). Spezifischer noch zur Anwendung des Boten-Konzepts auf Annotationen vgl. Jessen (in Vorbereitung). 
Man könnte schließlich sogar versuchen, das Verhältnis von lesender Person zu Text und Autor beziehungsweise Autorin als Machtverhältnis zu verstehen, als Machtverhältnis unter ungleichen Akteuren und Akteurinnen. Die Stiftspuren können dann auch Ausdruck einer Bewegung sein, die den Ausgleich eines asymmetrischen Machtverhältnisses sucht. So kann ein Text einer lesenden Person ein Wissen vermitteln, was umgekehrt nicht in der gleichen Form möglich ist. Ganz machtlos ist die lesende Person aber eben auch nicht: Weiß die lesende Person es besser, hat sie mit dem Stift ein kleines Machtausgleichsinstrument, mit dem sie einen Text immerhin korrigieren kann. Oder deren Autor als Propagandisten bloßstellen. Und sei es nur am Rande.

\section{Literaturverzeichnis}

\section{Archivmaterialien}

Mann, Thomas. Ausgewählte Erzählungen. Berlin: Aufbau. Originalstandort der Archivalie: Thomas-Mann-Archiv der ETH Zürich, Signatur: Thomas Mann 114. Digitalisate verfügbar in der Datenbank Thomas Mann Nachlassbibliothek. 1954.

Schaeder, Hans H. Goethes Erlebnis des Ostens. Leipzig: Hinrichs. Originalstandort der Archivalie: Thomas-Mann-Archiv der ETH Zürich, Signatur: Thomas Mann 552. Digitalisate verfügbar in der Datenbank Thomas Mann Nachlassbibliothek. 1938.

\section{Forschungsliteratur}

„annotate.“ In: T. F. Hoad (Hrsg.), The Concise Oxford Dictionary of English Etymology. Oxford: Oxford University Press. 1996.

„Note.“In: Wolfgang Pfeifer (Hrsg.), Etymologisches Wörterbuch des Deutschen, 2. Aufl. Berlin: Akademie Verlag. 1993, S. 933.

„notieren.“In: Elmar Seebold (Hrsg.), Kluge: Etymologisches Wörterbuch der deutschen Sprache. Berlin, Boston: De Gruyter. 2011, S. 660.

Atze, Marcel. Libri annotati: Annäherung an eine vernachlässigte Spezies: Hand- und Arbeitsexemplare. In: Marcel Atze und Volker Kaukoreit (Hrsg.), Lesespuren - Spurenlesen: Wie kommt die Handschrift ins Buch? Wien: Praesens. 2011, S. 11-51.

Bamert, Manuel. Gelesenes Gedrucktes: Textzentrierte Erklärungsansätze zur Entstehung von Lesespuren. In: Anke Jaspers und Andreas Kilcher (Hrsg.), Randkulturen. Göttingen: Wallstein. (In Vorbereitung).

Bergmann, Rolf und Stefanie Stricker (Hrsg.). Die althochdeutsche und altsächsische Glossographie: Ein Handbuch. Berlin: De Gruyter. 2009.

Bukauskaitė, Dalia. Kommentierter Katalog der nachgelassenen Bibliothek von Johannes Bobrowski. Trier: WVT Wissenschaftlicher Verlag Trier. 2006.

Fischer, Martin. Schrift als Notation. In: Peter Koch und Sybille Krämer (Hrsg.), Schrift, Medien, Kognition: Über die Exteriorität des Geistes. Tübingen: Stauffenburg. 1997, S. 83-101. 
Fogli, Daniela, Giuseppe Fresta und Piero Mussio. On Electronic Annotation and Its Implementation. In: Maria F. Costabile (Hrsg.), Proceedings of the Working Conference on Advanced Visual Interfaces. New York: ACM. 2004, S. 98-102.

Hausendorf, Heiko, Wolfgang Kesselheim, Hiloko Kato und Martina Breitholz. Textkommunikation: Ein textlinguistischer Neuansatz zur Theorie und Empirie der Kommunikation mit und durch Schrift. Berlin, Boston: De Gruyter. 2017.

Huse, Ulrich. Marginalie. In: Ursula Rautenberg (Hrsg.), Reclams Sachlexikon des Buches: Von der Handschrift zum E-Book. Stuttgart: Reclam. 2015, S. 276.

Jessen, Caroline. „!?!!“: Esoterisch/Exoterisch: Annotationen von Karl Wolfskehl. In: Anke Jaspers und Andreas Kilcher (Hrsg.), Randkulturen. Göttingen: Wallstein. (In Vorbereitung).

Köppe, Tilmann. Literatur und Wissen: Zur Strukturierung des Forschungsfeldes und seiner Kontroversen. In: Tilmann Köppe (Hrsg.), Literatur und Wissen: Theoretisch-methodische Zugänge. Berlin, New York: De Gruyter. 2011, S.1-28.

Krämer, Sybille. Spuren, Graphé, Wissenskünste: Zur Episteme der Spur. In: Sandie Attia, Ingrid Streble, Nathalie Le Bouëdec und Alice Volkwein (Hrsg.), Der Spur auf der Spur. Heidelberg: Universitätsverlag Winter. 2016, S. 19-30.

Maik, Philipp. Schreibstrategien und Leseverstehen. In: Ursula Rautenberg und Ute Schneider (Hrsg.), Lesen: Ein interdisziplinäres Handbuch. Berlin, Boston: De Gruyter. 2015, S. 207-232.

Mann, Thomas. Tagebücher 1918-1921. Herausgegeben von Peter de Mendelssohn. Frankfurt am Main: S. Fischer. 1979.

Mann, Thomas. Tagebücher 1937-1939. Herausgegeben von Peter de Mendelssohn. Frankfurt am Main: S. Fischer. 1980.

Mann, Thomas. Tagebücher 1944-1.4.1946. Herausgegeben von Inge Jens. Frankfurt am Main: S. Fischer. 1986.

Mann, Thomas. Lotte in Weimar: Kommentar. Große kommentierte Frankfurter Ausgabe 9.2. Herausgegeben von Werner Frizen. Frankfurt am Main: S. Fischer. 2003.

Martus, Steffen und Carlos Spoerhase. Praxeologie der Literaturwissenschaft. In: Geschichte der Germanistik 35/36. Göttingen: Wallstein. 2009, S. 89-96.

McCarty, Willard: Making and Studying Notes. Towards a Cognitive Ecology of Annotation. In: Julia Nantke und Frederik Schlupkothen (Hrsg.), Annotations in Scholarly Editions and Research. Functions, Differentiation, Systematization. Berlin, Boston: De Gruyter 2020, S. 271-297.

Moulin, Claudine. Am Rande der Blätter: Gebrauchsspuren, Glossen und Annotationen in Handschriften und Büchern aus kulturhistorischer Perspektive. In: Quarto. Zeitschrift des Schweizerischen Literaturarchivs 30/31. Genf: Editions Slatkine. 2010, S.19-26.

O’Hara, Kenton P., Alex Taylor, William Newman und Abigail J. Sellen. Understanding the Materiality of Writing From Multiple Sources. In: International Journal of Human-Computer Studies 56.3. Amsterdam: Elsevier. 2002, S. 269-305.

Ovsiannikov, Ilia A., Michael A. Arbib und Thomas H. McNeill. Annotation Technology. In: International Journal of Human-Computer Studies 50. Amsterdam: Elsevier. 1999, S. 329-362.

Wolff, Stephan. Wie kommt die Praxis zu ihrer Theorie? Über einige Merkmale praxissensibler Sozialforschung. In: Herbert Kalthoff, Stefan Hirschauer und Gesa Lindemann (Hrsg.), Theoretische Empirie: Zur Relevanz qualitativer Forschung. Frankfurt am Main: Suhrkamp. 2008, S. 234-259. 


\section{Weblinks}

Center for Digital Humanities at Princeton. Derrida's Margins. 2018. URL: https://derridasmargins.princeton.edu/ (14.10.2019).

Meister, Jan C. Glossar. 2016. URL: https://fortext.net/ressourcen/glossar (14.10.2019).

Olsen-Smith, Steven, Peter Norberg und Dennis C. Marnon. The Online Catalog of Books and Documents Owned, Borrowed and Consulted by Herman Melville. 2008. URL: http:// melvillesmarginalia.org/ (14.10.2019).

Theodor-Fontane-Archiv Potsdam und UCLAB an der Fachhochschule Potsdam. Fontanes Handbibliothek. 2019. URL: https://uclab.fh-potsdam.de/ff/ (14.10.2019).

Thomas-Mann-Archiv der ETH Zürich. Bibliotheksbestände und Nachlassbibliothek. 2019. URL: https://tma.ethz.ch/archiv/bestaende/bibliotheksbestaende-und-nachlassbibliothek.html (14.10.2019). 